Relations industrielles

Industrial Relations

\title{
Brown, Richard K., Understanding Industrial Organisations : Theoritical Perspectives in Industrial Sociology
}

\section{Denis Harrisson}

Volume 49, numéro 4, 1994

Syndicats et restructuration économique

Unions and Economic Restructuring

URI : https://id.erudit.org/iderudit/050983ar

DOI : https://doi.org/10.7202/050983ar

Aller au sommaire du numéro

Éditeur(s)

Département des relations industrielles de l'Université Laval

ISSN

0034-379X (imprimé)

1703-8138 (numérique)

Découvrir la revue

Citer ce compte rendu

Harrisson, D. (1994). Compte rendu de [Brown, Richard K., Understanding Industrial Organisations : Theoritical Perspectives in Industrial Sociology].

Relations industrielles / Industrial Relations, 49(4), 865-868.

https://doi.org/10.7202/050983ar

Tous droits réservés (C) Département des relations industrielles de l'Université Laval, 1994
Ce document est protégé par la loi sur le droit d'auteur. L'utilisation des services d'Érudit (y compris la reproduction) est assujettie à sa politique d'utilisation que vous pouvez consulter en ligne.

https://apropos.erudit.org/fr/usagers/politique-dutilisation/ 


\section{Understanding Industrial Organisations: Theoritical Perspectives in Industrial} Sociology, by Richard K. Brown, London and New-York, Routledge, 1992, 280 p., ISBN 0-415-01781-5 et 0-415-01782-3 (pbk).

Richard K. Brown est l'un des chefs de file de la sociologie industrielle britannique. Depuis près de trente ans, ses publications jalonnent la discipline sur des sujets aussi variés qu'érudits tels que la participation, le conflit et le changement dans l'entreprise, les attitudes au travail, les femmes et le travail, la stratification sociale et les relations industrielles, la flexibilité, les consultants en industrie. Ses travaux font preuve d'une grande maîtrise des apports empiriques et du développement théorique du travail industriel. La rédaction d'un livre sur les contributions britanniques à l'édification théorique des connaissances en organisation industrielle lui convenait.

Depuis quelques années, plusieurs ouvrages traçant le bilan de la sociologie industrielle ont été édités. Tout nouveau volume qui s'ajoute à cet amoncellement se doit donc d'offrir au lecteur une perspective nouvelle ou encore un découpage singulier du champ. D'emblée, l'auteur définit l'objet et il en propose certaines limites. Il s'agit de définir et de comprendre les caractéristiques et les interactions sociales des organisations industrielles. Mais le domaine est vaste et il apparaît comme une collection disparate et désintégrée d'études sur les relations et les conflits dans l'organisation du travail. L'auteur reconnaît la difficulté de s'arrêter à une définition autre que pragmatique dans laquelle la relation d'emploi révèle l'aspect analytique original de la sociologie industrielle. Richard K. Brown distingue celle-ci de l'étude des relations industrielles dont l'objet concerne de façon particulière les institutions régulatoires du travail et de l'emploi. Il constate avec justesse que la sociologie industrielle ne couvre pas l'ensemble des facettes du travail industriel. En effet, sans y trouver quelque justification théorique que ce soit, les sociologues industriels ont consacré la majeure partie de leurs travaux aux grandes firmes plutôt qu'aux petites, à l'industrie manufacturière plutôt qu'au secteur des services, au travail manuel plutôt qu'au travail non manuel et au travail des hommes plutôt qu'à celui des femmes. Il y a certes quelques exceptions mais l'on constate que les lacunes commencent à peine à être comblées en ces domaines.

Dans l'ouvrage, seuls les travaux d'auteurs britanniques depuis 1945 sont considérés. Richard K. Brown explique l'émergence de la sociologie industrielle par la convergence de trois contextes de la période d'après-guerre : a) le contexte institutionnel par la création de centres de recherche et de départements universitaires initiant des travaux sur les organisations et le travail ; b) le contexte intellectuel par la force d'attraction analytique du fonctionnalisme et du développement parallèle de courants critiques; c) le contexte socio-économique par les pressions exercées pour trouver des solutions à des problèmes pratiques du travail. Afin de circonscrire l'essentiel du sujet, l'auteur procède à un découpage thématique. L'ouvrage est divisé en quatre thèmes théoriques qui couvrent l'ensemble des travaux faits en sociologie industrielle : 1-la pensée systémique, 2- la théorie de la contingence, 3- la théorie de l'action, 4- la théorie du procès de travail. Il s'agit moins de présenter toutes les recherches qui ont été faites dans chacun de ces champs respectifs que d'analyser les travaux majeurs qui ont particulièrement influencé le développement théorique. Chaque thème fait l'objet d'un chapitre dont la structure est identique. Il s'agit de présenter les auteurs britanniques les plus importants pour chaque thème, de montrer les prémisses théoriques de chaque thèse, d'en 
développer l'argument principal, de présenter les travaux qui lui ont donné naissance, d'en démontrer l'importance et l'influence sur le développement de la sociologie industrielle et, enfin, de faire connaître les critiques à ces approches qui amorcent le développement d'autres approches théoriques.

Le concept de système joue un rôle prédominant dans la compréhension des organisations industrielles dont l'une des caractéristiques est de rassembler une variété de ressources pour la réalisation d'un objectif. La pensée systémique trouve un fondement théorique dans le fonctionnalisme où les ensembles sociaux sont traités comme des unités différenciées interdépendantes. Deux métaphores ont toujours servi le systémisme, la première emprunte à la mécanique l'idée qu'un système fermé doit permettre l'équilibre de ses parties, la seconde emprunte à la biologie l'idée qu'un système ouvert doit se développer en équilibre dynamique en permettant l'adaptation des parties au changement externe. En Angleterre, les premiers travaux de sociologie industrielle s'inspirant du systémisme ont été effectués par W.H. Scott de l'université de Liverpool sur les rapports entre les structures formelle, informelle et occupationnelle. Ces dernières représentent une description partielle de la structure sociale, elles peuvent être traitées séparément pour des besoins d'analyse mais elles sont interreliées et constituent un système interdépendant. Les frontières entre ces systèmes ne sont pas claires, elles sont constamment réinterprétées et interpénétrées d'éléments internes et externes. Les travaux de Liverpool furent intéressants pour l'étude des processus internes de l'entreprise ainsi que des valeurs et limites de la dichotomie des structures internes. Oubliée depuis, cette approche systémique donnera naissance à une variante qui sera encore plus percutante en sociologie industrielle, les systèmes socio-techniques ouverts. Puisant dans les écrits de Von Bertalanfly une option théorique plus forte ici, cette approche propose comme thèse fondamentale que les systèmes internes que forment la technologie et les relations sociales doivent s'ajuster l'un à l'autre et à l'environnement externe. Le système sociotechnique est un cadre de référence servant à expliquer le comportement des entreprises et leurs membres. En 1963, Rice annonce déjà l'entreprise flexible qui répond rapidement aux pressions externes, contrôle par les résultats, préconise les communications rapides, rend responsables les personnes et intègre des groupes de travail autorégulés. Dans cette approche, les effets psychologiques et sociaux de la technologie sont atténués par une organisation du travail adaptée. Cependant les critiques du système sociotechnique questionnent la conciliation des efforts productivistes et la satisfaction des ouvriers. Les théoriciens des systèmes socio-techniques n'ont pas remis en cause les fondements économiques de la firme et de la technologie. Les difficultés y sont traitées en termes d'adaptation d'une partie du système aux autres. L'entreprise est réifiée, les buts deviennent ceux de l'entreprise et non ceux des membres. L'emphase est mise sur le consensus et l'intégration et non sur les conflits. Un modèle universel d'organisation est idéalisé.

La théorie de la contingence se propose d'étudier l'organisation du travail et son environnement selon trois niveaux conceptuels d'analyse : la structure de l'organisation et son fonctionnement, la composition des groupes et l'interaction, la personnalité individuelle et son comportement. Le projet est d'une grande envergure. Alors que l'approche précédente trouve sa légitimité dans des études de cas, ici les méthodes quantitatives objectivistes et positivistes sont préconisées. La théorie de la contingence puise ses fondements théoriques dans les écrits de Weber sur la bureaucratie, l'autorité et la règle 
de légitimité. Dans cette approche, l'explication des variations se trouve dans les réponses des organisations à certains stimuli. Pour Woodward, la relation entre la technologie et l'organisation semble déterminante de sa structure. Pour sa part, le groupe d'Aston tente d'expliquer la variation par le contexte de l'organisation, le climat organisationnel et le comportement des groupes et des individus. On y mesure l'effet de variables aussi diverses que la taille de l'entreprise, la technologie, la composition de la main-d'œuvre, le type de propriété, les valeurs de l'organisation, son histoire, etc. On découvre que la bureaucratie ne forme pas un ensemble unitaire mais qu'il y a plusieurs façons bureaucratiques déterminées par des variables internes et externes. Les critiques ont tôt fait de remarquer que le groupe d'Aston n'a pas utilisé les fondements théoriques de leurs concepts-clés. La liste des variables contextuelles est ad hoc et l'organisation reste mal définie. La théorie de la contingence implique un degré élevé de détermination de la structure organisationnelle par le contexte. Child ajoutera qu'il existe un choix d'arrangements structurels qui médiatisent les contraintes environnementales. Ce choix est influencé par l'idéologie des membres. Ici, l'agence humaine est réintroduite dans un débat qui s'est davantage préoccupé d'associations statistiques entre des caractéristiques organisationnelles. La théorie de la contingence survit dans une forme modifjée où elle cède néanmoins sous le poids de la variable «choix stratégique ». Cette notion est cependant problématique dans la mesure où peu d'études se sont réellement intéressées aux valeurs et aux orientations d'action des employés. Ce sont là les arguments qui serviront le prochain débat: l'approche de l'action.

L'approche systémique dépend de certaines suppositions psychologiques de base : les besoins des gestionnaires et la satisfaction des ouvriers. La théorie de la contingence implique aussi que les valeurs de certains membres sont cruciales. Mais on suppose dans un cas comme dans l'autre que ces comportements sont universels et que les variations observées sont dues à des variations de tâches ou aux rôles. Le projet de l'approche de l'action naît de la critique de l'homo economicus. L'ouvrier est en quête d'expression de soi, il cherche l'autonomie et le contrôle, un sens à son travail qui conduit à la satisfaction et à l'implication. Ici, la structure des organisations est le produit de l'action humaine. Les études de Goldthorpe proposent un cadre explicatif alternatif à l'approche des relations humaines et à la théorie de la contingence notamment celui développé par Woodward et le déterminisme technologique. Les orientations au travail sont aussi influencées par le non-travail et introduites dans l'organisation par les travailleurs. Ces derniers sont réalistes quant aux attentes du travail de sorte que leurs priorités demeurent très limitées conduisant ainsi à une « instrumentalisation aliénée » du travail. Influencé par les théories de Weber, Schultz et les ethnométhodologues, Silverman propose pour sa part une méthode d'analyse qui repose sur les définitions des acteurs euxmêmes en référence aux ressources stratégiques dont ils disposent. L'approche de l'action veut compléter l'approche systémique, contrairement à cette dernière elle laisse entrevoir des conflits compte tenu des intérêts divergents des groupes qui composent l'organisation. Mais cette approche est loin de satisfaire ceux qui voient un conflit majeur entre le capital et le travail indépendamment de ce qu'en pense l'acteur. Ces critiques annoncent un débat sur le procès de travail et le pouvoir ouvrier.

La théorie du procès de travail a ouvert de nouvelles perspectives théoriques dans la sociologie industrielle britannique notamment par les analyses de l'organisation du travail, des qualifications, du contrôle du travail. Inspiré par Marx pour les relations 
entre le capital et le travail, et Braverman pour le procès de déqualification du travail par l'organisation scientifique du travail, on y affirme que les changements dans l'organisation sont toujours limités dans leur portée et dans leurs effets. L'organisation est analysée dans un contexte local, national et international où le pouvoir du travail est produit et reproduit sur une base quotidienne. L'organisation du travail ne peut être isolée de ses déterminants sociétaux. Le projet consiste à comprendre le capitalisme et ses modalités de contrôle (Friedman), le consentement et l'acceptation des règles dans le régime de l'entreprise (Burawoy), les qualifications (Littler, Manwaring et Wood). Ce faisant, l'approche laisse en plan des dimensions inexplorées qui remettent en question l'affirmation d'une théorie du procès de travail capitaliste, soit un véritable pattern capitaliste de dégradation du travail dans lequel la thèse même du procès de déqualification à coup de changements successifs dans le contrôle du travail, la perte d'autonomie des ouvriers, le développement technologique ne tient plus.

Partant de ces quatre théories, on en retire deux types de sociologie apparemment incompatibles : l'un qui s'intéresse aux principes structuraux de la régulation des relations entre employeurs et employés; l'autre qui analyse la structure comme un ordre négocié portant peu attention aux contraintes externes. Cependant il y a complémentarité des approches dans la mesure où chacune apporte une contribution dans le débat continu des organisations et de la sociologie industrielle. Il y a ainsi une pluralité de systèmes au sein d'une même organisation, les configurations de celle-ci sont le résultat de l'action qui est elle-même influencée par des variables structurelles et les contextes socioéconomique, politique et technologique. Enfin, nul ne peut ignorer les conflits d'intérêts inéluctables entre les agents dans une même organisation. Le contrôle des salariés et les moyens développés pour contrer les conséquences contradictoires qui en résultent derneurent le problème-clé des organisations. En bref, il n'existe pas de synthèse théorique qui puisse embrasser les quatre théories, l'organisation industrielle étant un ensemble complexe. Ces quatre grandes théories sont nécessaires à notre compréhension des organisations mais elles sont peu utiles à l'interprétation des changements en cours dans les firmes industrielles. Sans vouloir prédire l'avenir, Richard K. Brown voit dans la flexibilité une notion centrale à partir de laquelle peut se créer de nouveaux modèles théoriques, de nouveaux concepts pour intégrer les transformations dans la composition de la main-d'œuvre, la structure distributive de l'emploi de même que l'organisation et la gestion du travail rémunéré. La flexibilité est considérée comme un idéal-type donnant lieu à de nouvelles controverses sur les solutions de rechange au taylorisme comme base d'organisation et de contrôle à l'aube du XXI' siècle.

Ce livre est bien fait et bien écrit. Les quatre théories aussi dissemblables soientelles y sont clairement exposées avec la même rigueur et la même érudition. En faisant le choix de quelques auteurs et de leur thèse principale, les différentes théories exposées peuvent être approfondies davantage et aisément comprises du lecteur. Ce volume est recommandé pour quiconque s'intéresse à la sociologie du travail et à l'étude des organisations.

Denis Harrisson 